\title{
UMBU: Um Framework para Experimentação e Avaliação de QoE em Transmissões Multimídia
}

\author{
Carlos H. de Azevedo ${ }^{1}$, Emídio P. Neto ${ }^{1}$, Charles H. F. dos Santos ${ }^{1}$ \\ Felipe S. Dantas Silva ${ }^{1,2}$, Augusto Venâncio Neto ${ }^{2,3}$
}

\author{
${ }^{1}$ Laboratório de Tecnologias Avançadas em Redes de Computadores (LaTARC) \\ Instituto Federal de Educação, Ciência e Tecnologia do Rio Grande do Norte (IFRN) \\ Natal-RN, Brasil \\ ${ }^{2}$ Departamento de Informática e Matemática Aplicada (DIMAp) \\ Universidade Federal do Rio Grande do Norte (UFRN) - Natal-RN, Brasil \\ ${ }^{3}$ Instituto de Telecomunicações (IT), Aveiro, Portugal \\ \{carlos.h, emidio.neto\}eacademico.ifrn.edu.br \\ \{charles.hallan, felipe.dantas\}@ifrn.edu.br, augusto@dimap.ufrn.br
}

\begin{abstract}
Among the leading available solutions for network multimedia Quality of Experience (QoE) analysis, EvalVid is the primary open source reference in this area. However, this tool has numerous shortcomings ranging from the installation to QoE analysis, imposing severe restrictions that limit the operationalization of large-scale evaluations. In view of this, this paper proposes a breakthrough in the multimedia transmission QoE experimentation field, the Unified Multimedia-based Network Evaluation Platform (UMBU), a framework for multimedia experimentation and evaluation featuring a ubiquitous interface for refined management of experiments-related operations, as well as to provide a series of mechanisms that allow the conduction of multiple assessments in a scalable and flexible way.
\end{abstract}

Resumo. Dentre as principais soluções disponíveis para análise de Qualidade de Experiência (QoE) em transmissões multimídia, o EvalVid se apresenta como a principal referência em código aberto dessa área. No entanto, esta ferramenta possui inúmeras deficiências, que vão desde a instalação até a análise do QoE, impondo sérias restrições que limitam a operacionalização de avaliações em larga escala. Em vista disso, este trabalho propõe um avanço no campo da experimentação de QoE em transmissões multimídia através do Unified Multimedia-based Network Evaluation Platform (UMBU), um framework para experimentação e avaliação cujo objetivo é fornecer uma interface que possibilita acesso ubíquo para o gerenciamento refinado sobre as operações relacionadas aos experimentos, além de prover uma série de mecanismos que possibilitam a realização de múltiplas avaliações de maneira escalável e flexível.

\section{Introdução}

O aumento da demanda e oferta sobre os serviços disponibilizados por meio da atual infraestrutura da Internet evidenciou a insuficiência de recursos e mecanismos para satisfazer as novas expectativas dos usuários, bem como prover suporte aos novos re- 
quisitos das aplicações. Desta maneira, diversas alternativas que se mostraram potencialmente capazes de viabilizar o desenvolvimento de novas soluções para infraestruturas de redes foram empregadas, a exemplo dos novos arcabouços tecnológicos baseados no paradigma de Redes Definidas por Software (Software-Defined Networking SDN) [Boucadairm and Jacquenet 2014] e na Virtualização de Funções de Rede (Network Function Virtualization - NFV) [Mijumbi et al. 2016]. Além disso, novos modelos de distribuição de conteúdo, como Computação em Nuvem e arquiteturas para suporte a mobilidade baseadas em arquiteturas de Redes sem Fio para Objetos Móveis (Wireless Networks for Moving Objects - WiNeMO) [Silva et al. 2016] também foram largamente empregadas para suportar a crescente demanda dos usuários.

Neste contexto, é esperado que novas estratégias sejam desenvolvidas e implantadas com o intuito de avaliar a eficácia dessas novas soluções, medindo o desempenho e eficiência da infraestrutura em diferentes parâmetros e sob variadas circunstâncias. Dentre as principais maneiras de se verificar o desempenho da rede estão as análises da satisfação do usuário sobre um determinado serviço (aplicação), expresso por meio de métricas de Qualidade de Experiência (Quality of Experience - QoE) [Chen et al. 2015]. Visto que o volume de tráfego das aplicações multimídia (e.g., vídeo sob demanda) se tornou majoritário na Internet nos últimos anos [Cisco 2017], a análise de QoE em aplicações multimídia se tornou fundamental para aferir a aceitabilidade geral do sistema.

Medições tradicionais dos níveis de QoE consistem na realização de vários experimentos onde os avaliadores indicam subjetivamente as suas percepções sobre o conteúdo disponibilizado, utilizando-se de um sistema de pontuação baseado em níveis (e.g., ruim, regular, bom) de opinião (e.g., Mean Opinion Score - MOS) [Clark et al. 2014]. Embora possua resultados precisos, esse tipo de aferição de QoE depende de vários fatores restritivos, como disponibilidade financeira, condições de experimentação e esforço laboral, além de não se tratar de um método capaz de fornecer resultados em tempo real, inviabilizando avaliações em larga escala.

Em vista disso, modelos computacionais foram desenvolvidos com o objetivo de aferir a fidelidade da sequência de imagens recebidas por serviços multimídia, de modo a medir, de maneira objetiva, o impacto o desempenho da rede na perspectiva da experiência do usuário. Amplamente utilizados pela comunidade científica, esses métodos consistem em modelos que se baseiam no Sistema Visual Humano (Human Visual System - HVS) para captar informações dos sinais luminosos das imagens, sendo assim utilizados para medir quantitativamente a experiência do usuário [Wang et al. 2003]. São exemplos de métricas objetivas o Peak Signal-to-Noise Ratio (PSNR), Structural Similarity (SSIM) e o Video Quality Metric (VQM) [Chikkerur et al. 2011].

A condução da avaliação de QoE em transmissões multimídia geralmente é realizada através de alguma ferramenta que operacionalize a extração das métricas a partir do vídeo processado. O Evalvid [Klaue et al. 2003], atualmente a principal instância nesse segmento, consiste em uma solução de código aberto capaz de abranger diversos aspectos das avaliações, que compreende a preparação da mídia, envio do conteúdo através da rede, captura do conteúdo no destino, reconstrução do vídeo capturado e geração das métricas de QoE. Embora disponibilizado com um conjunto de ferramentas e funcionalidades para a execução dos experimentos, o Evalvid apresenta problemas que afetam a flexibilidade e escalabilidade em procedimentos de avaliações de QoE, principalmente 
quanto ao envio simultâneo de várias sessões para distintos clientes, cada uma com requisitos de tráfego diferentes. Além disso, esses procedimentos requerem a entrada manual de diversos parâmetros, aumentando a possibilidade de erros humanos e afetando a produtividade geral das avaliações. Um outro aspecto restritivo se refere à limitação das métricas disponíveis para aferição de QoE, requerendo para isso o uso de ferramentas adicionais (e.g., MSU VQMT ${ }^{1}$ ) que possuam uma maior variedade de modelos de avaliação, de modo a otimizar os níveis de precisão sobre as medidas de qualidade do conteúdo. No tocante ao apoio de novos usuários, a quase inexistente documentação sobre os seus procedimentos dificulta ainda mais o entendimento geral de seu funcionamento.

Em vista disso, este trabalho propõe o Unified Multimedia-based Network Evaluation Platform (UMBU), um framework cujo objetivo é fornecer uma plataforma de acesso ubíquo para automatização dos processos de avaliação de QoE em transmissões multimídia. Para alcançar esse objetivo, UMBU foi projetado para superar as dificuldades introduzidas pelo Evalvid ao disponibilizar interfaces interativas responsáveis por auxiliar as operações referentes às avaliações de QoE, que compreendem a instalação do framework e seus pré-requisitos, preparação, transmissão e reconstrução da mídia, além de estender as possibilidades de métricas de QoE para extração e obtenção dos resultados em formato de gráficos. Além disso, UMBU provê um conjunto de funcionalidades que possuem a finalidade de prover uma plataforma flexível e escalável de experimentação, como paralelismo de avaliações e organização hierárquica e dinâmica de projetos e experimentos. UMBU possui o objetivo de atender uma ampla variedade de plataformas, não sendo restrito à simuladores/emuladores em particular, sendo, desta maneira, possível de ser executado em ambientes reais.

O restante deste trabalho está estruturado da seguinte maneira: a Seção 2 apresenta um estudo em trabalhos relacionados para destacar a contribuição do UMBU; a Seção 3 fornece uma descrição detalhada do UMBU, incluindo sua arquitetura, componentes principais, operações e interfaces; a Seção 4 detalha a proposta de demonstração da ferramenta; a Seção 5 fornece considerações finais e faz apontamentos para trabalhos futuros.

\section{Trabalhos Relacionados}

Várias ferramentas foram propostas no campo de avaliação de qualidade multimídia com o objetivo de prover as capacidades necessárias em medir os níveis de QoE dos conteúdos transmitidos. Dentre as principais ferramentas, estão incluídas soluções baseadas no software de computação numérica $\mathrm{MATLAB}^{2}$, como o MetriX MuX Visual Quality Assessment Package [Gaubatz and Hemami 2011], que provê 12 (doze) métricas para avaliação de qualidade das imagens. No entanto, essa solução não é especializada em experimentações via rede, além de não possuir uma interface intuitiva, sendo basicamente um conjunto de funções que são chamadas a partir da linha de comando do MATLAB.

Os autores em [Murthy and Karam 2010] também propuseram uma solução de avaliação a partir do MATLAB, através de uma interface capaz de realizar a abstração de detalhes (como formatos de arquivos), bem como um arcabouço para a adição de novas métricas. A solução também provê um ambiente para avaliação subjetiva do conteúdo

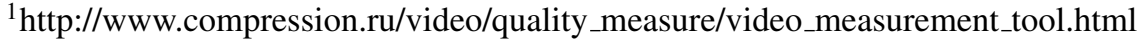

${ }^{2}$ https://www.mathworks.com/products/matlab.html
} 
multimídia, não possuindo, entretanto, métodos para automação de avaliações via rede. Uma revisão mais completa das propostas relacionadas baseadas no MATLAB pode ser encontrada em [Krasula et al. 2011].

Entre as ferramentas proprietárias, as que mais se destacam são o MSU Video Quality Measurement Tool (VQMT) [Vatolin et al. 2009] e o AccepTV Video Quality Analyzer (VQA) ${ }^{3}$. Suas principais funcionalidades incluem análises que oferecem suporte à múltiplos formatos de mídia, interfaces em linha de comando em Graphical User Interface (GUI) e suporte à resoluções de até 8K. O AccepTV VQA também oferece, além do ambiente de análise de QoE, uma interface web para extração e visualização das métricas. Embora se figurem como boas opções para análise de QoE, o valor de aquisição das licenças impõem uma restrição significativa na aquisição dos softwares, além de não fornecerem mecanismos para experimentação em redes de computadores.

Dentre as ferramentas disponíveis para análise de QoE multimídia, o Evalvid [Klaue et al. 2003] surge como uma solução especializada em operações relacionadas à transmissão de vídeos via rede, possuindo, entretanto, as limitações mencionadas anteriormente. Alguns trabalhos [Ke et al. 2006] [Ke et al. 2008] foram propostos para otimizar os modelos de experimentação baseados no Evalvid no que diz respeito à reprodução de erros, mas estão limitados ao simulador NS-2. Os autores em [Bouras et al. 2013] propuseram uma interface gráfica para o NS-2 cujas funcionalidades compreendem simulações baseadas no Evalvid. Atualmente, com o surgimento de outros simuladores e emuladores de rede, como o NS-3, GNS-3, Core e Mininet, a limitação dessas propostas ao NS-2 impõe uma restrição significativa à flexibilidade no uso do Evalvid.

Desse modo, a não ser pela GUI referenciada ${ }^{4}$ pelos autores do Evalvid, cujo repositório não disponibiliza o código-fonte (o que sugere que o projeto foi descontinuado), no melhor do nosso conhecimento, nenhuma outra solução foi proposta como um framework de gerenciamento de experimentações de QoE que é independente das plataformas de simulação e emulação, e que compreende a totalidade das operações de experimentação (i.e., instalação, extração de métricas e plotagem de resultados).

Nessa perspectiva, a próxima seção introduz o framework UMBU, que otimiza o processo de avaliação de QoE por meio de uma interface ubíqua que tem como objetivo dar suporte aos procedimentos de medição da qualidade multimídia, bem como fornecer uma estrutura que é capaz de otimizar a organização dos experimentos em sua totalidade.

\section{UMBU Framework}

A arquitetura do UMBU se apresenta como um framework integrador composto por módulos especializados em gerenciar um conjunto de ferramentas que abrangem o processo de avaliação de QoE via rede. UMBU, que disponibiliza uma interface única e amigável para gerenciamento das avaliações, também maximiza os níveis de organização dos experimentos no sistema de arquivos local, além de permitir a realização de avaliações simultâneas para múltiplos clientes distintos de forma centralizada. Tais funcionalidades não se fazem presentes nas ferramentas atualmente disponíveis, sendo uma das maiores limitações apresentadas por essas soluções.

\footnotetext{
${ }^{3}$ http://www.acceptv.com/en/products_vqa.php

${ }^{4}$ https://launchpad.net/evalvidgui
} 
A Figura 1 fornece uma visão geral sobre a arquitetura UMBU e seus módulos funcionais. UMBU atua em duas instâncias principais: ( $i$ ) servidor (responsável pela transmissão do vídeo); (ii) e cliente (que possui as funcionalidades para recepção da mídia).

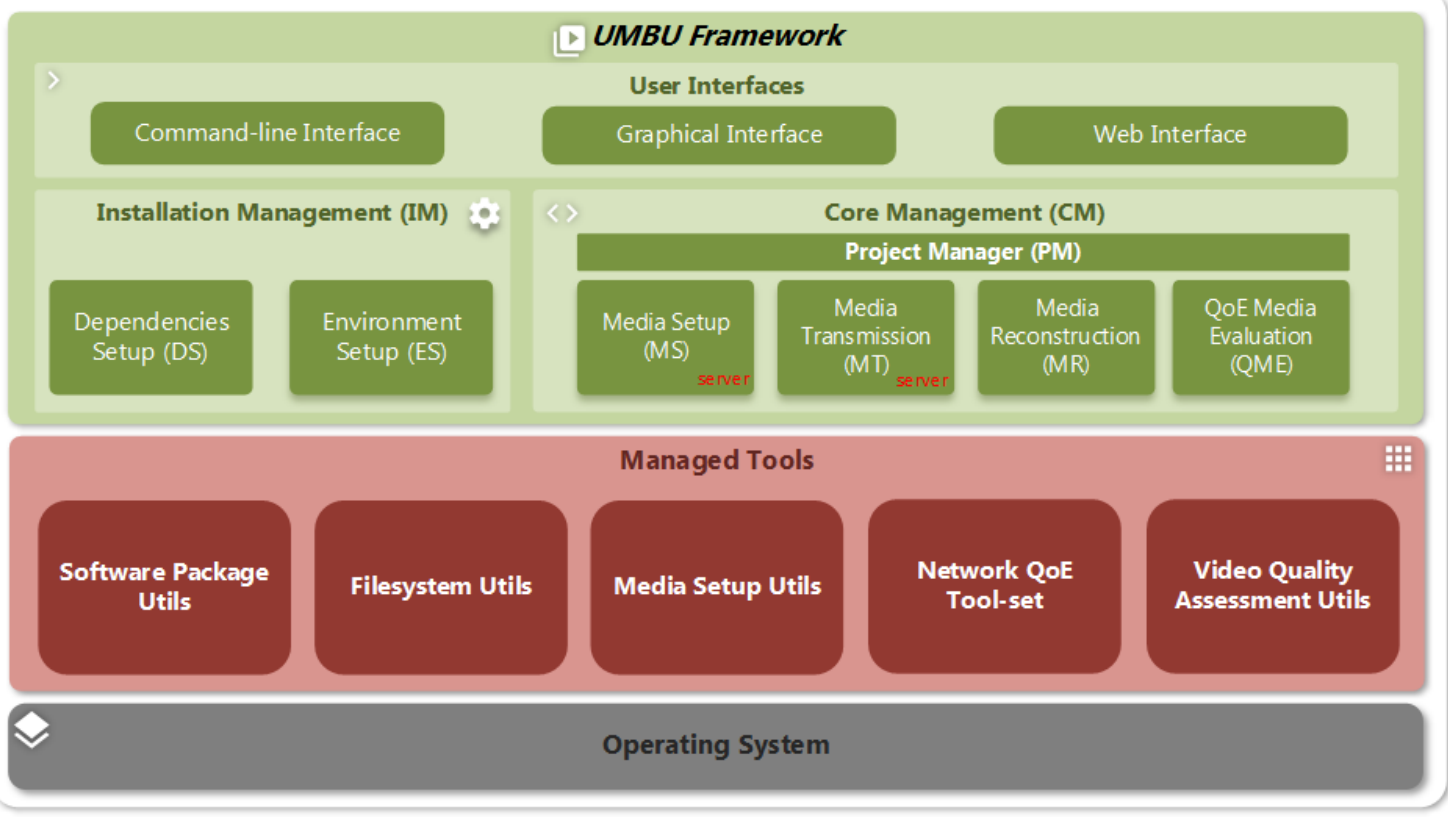

Figura 1. Arquitetura do framework UMBU

O framework consiste em três componentes principais: (i) interfaces do usuário, que podem consistir em um Command-line Interface (CLI), uma interface gráfica e uma plataforma web; (ii) o (Installation Management - IM), responsável por instalar as dependências das ferramentas e preparar o ambiente do usuário; e (iii) o (Core Management - CM), que contém os módulos responsáveis por gerenciar diretamente as ferramentas utilizadas. As subseções seguintes descrevem os principais componentes do framework, bem como seus módulos e funcionalidades.

\subsection{Installation Management (IM)}

O Installation Management (IM) é responsável por realizar todas as operações relacionadas à detecção, preparo e instalação de dependências que são necessárias para o funcionamento da ferramenta, além de configurar o ambiente para o uso (e.g., configuração da estrutura de diretórios no sistema de arquivos local). Para esse fim, o IM se baseia em dois módulos principais: (i) Dependencies Setup (DS); e (ii) Environment Setup (ES).

O DS gerencia as utilidades requeridas para a instalação das aplicações utilizadas pelo UMBU, bem como suas dependências. Para essa finalidade, o DS possui registros que mantém a identificação de todas as ferramentas e bibliotecas requeridas para o funcionamento do framework. Esse módulo provê compatibilidade com um conjunto de distribuições baseadas em Linux, como o Debian e Ubuntu, tomando como base a arquitetura do sistema operacional (e.g., i386, x64) para a localização dos recursos apropriados.

O ES, por sua vez, é responsável pela preparação do ambiente de uso, gerando uma estrutura hierárquica de diretórios para a organização de vários projetos que englobam um 
número de experimentos. UMBU provê a devida organização das mídias e das métricas geradas, conforme descrito nas seções seguintes.

\subsection{Core Management $(\mathbf{C M})$}

O Core Management (CM) é o componente responsável por concentrar as principais funcionalidades utilizadas durante a execução do UMBU. Este componente contém módulos cujas funções abrangem as principais funções do framework: (i) preparo (Media Setup MS); (ii) transmissão (Media Transmission - MT); (iii) captura e reconstrução da mídia (Media Reconstruction - MR); e (iv) extração de métricas (QoE Media Evaluation QME). O modo de operação desses módulos está condicionado à função das instâncias de execução do framework (i.e., servidor ou cliente).

Além disso, esses módulos são governados por um gerenciador de projetos (Project Manager - PM), que é responsável por realizar a gerência dos diferentes ambientes de experimentação nas quais a ferramenta terá de lidar. Com base nisso, uma breve descrição de cada módulo é fornecida a seguir:

- Project Manager (PM) - Responsável por criar vários ambientes (projetos) de experimentação de QoE, organizados em um conjunto de diretórios no sistema de arquivos. Cada projeto contém sua própria coleção de mídias, sendo também organizado em uma estrutura de experimentos com diretórios dedicados, possuindo seus próprios resultados para finalidades de comparação e análise posteriores.

- Media Setup (MS) - Essa função do servidor é responsável pela configuração do conteúdo antes da transmissão, sendo compatível com mídias de origem de diferentes formatos (e.g., MP4, YUV, 264). Ao receber a mídia fornecida pelo usuário, o MS aciona as ferramentas responsáveis pela conversão da mídia para o formato apropriado de envio.

- Media Transmission (MT) - Responsável por realizar os procedimentos necessários para a transmissão da mídia (através do protocolo RTP) após a preparação realizada pelo MS.

- Media Reconstruction (MR) - Possui a função de capturar as informações sobre pacotes transmitidos pelo servidor, bem como os pacotes recebidos pelo cliente UMBU. Ao término desse processo, deve ser gerado um vídeo que reconstitui a qualidade da mídia conforme recebido pelo cliente.

- QoE Media Evaluation (QME) - Utilizando a mídia gerada pelo MR, o QME possui a funcionalidade de gerenciar as ferramentas responsáveis pela geração de métricas de QoE mais comuns (e.g., VQM, SSIM, VIFp). As principais ferramentas utilizadas para esse fim são o Vooya ${ }^{5}$ e o MMSPG VQMT ${ }^{6}$, que avaliam a qualidade da mídia com base em uma referência (i.e., vídeo transmitido), que é coletada com base nas mídias geradas pelo MS, indexando os valores referentes à qualidade indicados pelas métricas. Além disso, este módulo provê a funcionalidade de geração de gráficos para melhor visualização dos resultados.

\section{Demonstração}

Maiores detalhes sobre o UMBU (código-fonte, funcionamento básico e manuais de instalação e utilização, além de um vídeo da ferramenta em funcionamento) podem ser

\footnotetext{
${ }^{5}$ http://www.offminor.de/

${ }^{6} \mathrm{https}: / / \mathrm{mmspg} . \mathrm{epfl} . \mathrm{ch} / \mathrm{vqmt}$
} 
encontrados no repositório oficial do projeto $^{7}$. Um ambiente baseado em máquinas virtuais pré-configuradas também está disponível, de modo a facilitar o uso da ferramenta por novos usuários.

A demonstração consiste em apresentar as funcionalidades atualmente disponíveis no UMBU para realização de experimentações de QoE em transmissões multimídia e, para tal, serão considerados dois casos de uso:

- Caso 1 - Serão demonstradas as operações básicas do framework para realização de experimentos simples. Neste caso, serão utilizadas tanto mídias que já são disponibilizadas com a ferramenta, quanto demais sequências de mídias obtidas a partir da Internet. Neste experimento serão utilizados simuladores/emuladores como o NS-3 e Mininet, amplamente utilizados pela comunidade científica, e máquinas virtuais, configuradas em hosts físicos distintos, a fim de demonstrar a capacidade de funcionamento em ambientes heterogêneos.

- Caso 2 - Será demonstrado a utilização do UMBU no testbed FIBRE. A fim de expor seu potencial de escalabilidade, serão considerados cenários de transmissão $n$ para $n$, onde múltiplas sessões multimídia serão transmitidas para clientes distintos, com requisitos de qualidade diversos, simultaneamente. Desta maneira, será confirmada a eficácia do UMBU ao eliminar a necessidade da configuração de tráfego sintético com ferramentas como Iperf ${ }^{8}$, por exemplo, atualmente uma das manobras técnicas mais utilizadas para a criação de tráfego concorrente.

\section{Conclusão}

Neste trabalho propomos o UMBU, um framework de apoio à experimentação e avaliação de QoE em transmissões multimídia. Ao melhor do nosso conhecimento, confirmado por estudos em trabalhos relacionados, não há evidências de propostas anteriores que forneçam suporte à análise de QoE em transmissões multimídia de larga escala e com acesso ubíquo, de modo a abranger a maior parte possível dos processos de avaliação (desde a instalação da ferramenta até a geração de resultados), conforme proposto pelo framework aqui introduzido.

UMBU é capaz de abstrair a complexidade referente à realização de experimentos em larga escala por meio da sua arquitetura, que permite que novos recursos sejam implementados sem a necessidade de alteração das funcionalidades dos seus componentes, também permitindo a compatibilidade com diferentes plataformas de simulação/emulação de sistemas em rede, bem como contextos em cenários reais. Os próximos passos referentes ao desenvolvimento desta ferramenta consistem no acréscimo de novas ferramentas e métricas, de modo a maximizar as capacidades de gerenciamento providas pelo framework.

\section{Referências}

Boucadairm, M. and Jacquenet, C. (2014). Software-Defined Networking: A Perspective from within a Service Provider Environment. Internet Engineering Task Force Request for Comments (RFC 7149).

\footnotetext{
${ }^{7}$ https://latarc.ifrn.edu.br/umbu/

${ }^{8}$ https://iperf.fr/
} 
Bouras, C., Charalambides, S., Drakoulelis, M., Kioumourtzis, G., and Stamos, K. (2013). Simulation design and execution-the case of trafil. In SIMULTECH, pages 27-34.

Chen, Y., Wu, K., and Zhang, Q. (2015). From QoS to QoE: A tutorial on video quality assessment. IEEE Communications Surveys \& Tutorials, 17(2):1126-1165.

Chikkerur, S., Sundaram, V., Reisslein, M., and Karam, L. J. (2011). Objective video quality assessment methods: A classification, review, and performance comparison. IEEE transactions on broadcasting, 57(2):165-182.

Cisco, C. V. N. I. (2017). Global mobile data traffic forecast update, 2016-2021 white paper, 2017.

Clark, A., Wu, Q., Schott, R., and Zorn, G. (2014). RTP Control Protocol (RTCP) Extended Report (XR) Blocks for Mean Opinion Score (MOS) Metric Reporting. Internet Engineering Task Force Request for Comments (RFC 7266).

Gaubatz, M. and Hemami, S. (2011). Metrix mux visual quality assessment package. http://www.foulard.ece.cornell.edu/gaubatz/metrix_mux.

Ke, C.-H., Lin, C.-H., Shieh, C.-K., and Hwang, W.-S. (2006). A novel realistic simulation tool for video transmission over wireless network. In Sensor Networks, Ubiquitous, and Trustworthy Computing, 2006. IEEE International Conference on, volume 1, pages 7-pp. IEEE.

Ke, C.-H., Shieh, C.-K., Hwang, W.-S., and Ziviani, A. (2008). An evaluation framework for more realistic simulations of mpeg video transmission. Journal of Information Science \& Engineering, 24(2).

Klaue, J., Rathke, B., and Wolisz, A. (2003). Evalvid-a framework for video transmission and quality evaluation. In International Conference on Modelling Techniques and Tools for Computer Performance Evaluation, pages 255-272. Springer.

Krasula, L., Klíma, M., Rogard, E., and Jeanblanc, E. (2011). Matlab-based applications for image processing and image quality assessment-part i: Software description. Radioengineering, 20(4):1009-1015.

Mijumbi, R., Serrat, J., Gorricho, J. L., Bouten, N., Turck, F. D., and Boutaba, R. (2016). Network function virtualization: State-of-the-art and research challenges. IEEE Communications Surveys Tutorials, 18(1):236-262.

Murthy, A. V. and Karam, L. J. (2010). A matlab-based framework for image and video quality evaluation. In Quality of Multimedia Experience (QoMEX), 2010 Second International Workshop on, pages 242-247. IEEE.

Silva, F. S. D., Neto, A. V., Maciel, D., Castillo-Lema, J., Silva, F., Frosi, P., and Cerqueira, E. (2016). An innovative software-defined winemo architecture for advanced qos-guaranteed mobile service transport. Computer Networks, 107:270-291.

Vatolin, D., Moskvin, A., Petrov, O., and Trunichkin, N. (2009). MSU Video Quality Measurement Tool.

Wang, Z., Sheikh, H. R., Bovik, A. C., et al. (2003). Objective video quality assessment. The handbook of video databases: design and applications, 41:1041-1078. 